\title{
STANDARD COMPONENTS OF A KRULL-SCHMIDT CATEGORY
}

\author{
SHIPING LIU AND CHARLES PAQUETTE \\ (Communicated by Birge Huisgen-Zimmermann) \\ In memory of Michael C. R. Butler
}

\begin{abstract}
We provide criteria for an Auslander-Reiten component having sections of a Krull-Schmidt category to be standard. Specializing to the category of finitely presented representations of a strongly locally finite quiver and its bounded derived category, we obtain many new types of standard Auslander-Reiten components. An application to the module category of a finite-dimensional algebra yields some interesting new results.
\end{abstract}

\section{INTRODUCTION}

Standard Auslander-Reiten components of the module category of a finitedimensional algebra are extremely interesting, since the maps between modules in such a component can be described in a simple combinatorial way; see [4, 13. This kind of component appears mainly for representation-finite algebras, hereditary algebras, tubular algebras and tilted algebras (see [13]), and each of them has at most finitely many non-periodic Auslander-Reiten orbits; see [14. In particular, the regular ones are stable tubes or of shape $\mathbb{Z} \Delta$ with $\Delta$ a finite acyclic quiver.

On the other hand, the Auslander-Reiten theory has been extended to KrullSchmidt categories; see [2,11. It is natural to expect that new types of standard Auslander-Reiten components will appear in this context. Indeed, in the most general setup, we shall find various criteria for such an Auslander-Reiten component having sections to be standard. In particular, an Auslander-Reiten component which is a wing or of shape $\mathbb{N A}_{\infty}^{+}, \mathbb{N}^{-} \mathbb{A}_{\infty}^{-}$or $\mathbb{Z} \mathbb{A}_{\infty}$ is standard if and only if its quasisimple objects are pairwise orthogonal bricks. Specializing to $\operatorname{rep}^{+}(Q)$, the category of finitely presented representations of a connected strongly locally finite quiver $Q$, we prove that the preprojective component and the preinjective components are standard, and every component is standard in case $Q$ is of finite or infinite Dynkin type. Applying this to the bounded derived category $D^{b}\left(\operatorname{rep}^{+}(Q)\right)$ of $\operatorname{rep}^{+}(Q)$, we show that the connecting component is standard and that every component is standard in the finite or infinite Dynkin case. These results imply particularly the

\footnotetext{
Received by the editors August 24, 2012 and, in revised form, January 29, 2013 and March 6, 2013.

2010 Mathematics Subject Classification. Primary 16G70, 16G20, 16G10.

Key words and phrases. Krull-Schmidt categories, almost split sequences, Auslander-Reiten quiver, standard components, representations of quivers, tilted algebras.

The first-named author was supported in part by the NSERC of Canada.

The second-named author was supported in full by NSERC and AARMS.
} 
existence of standard Auslander-Reiten components which are wings or of shapes $\mathbb{N A}_{\infty}^{+}, \mathbb{N}^{-} \mathbb{A}_{\infty}^{-}$and $\mathbb{Z} \Delta$, where $\Delta$ is an arbitrary strongly locally finite quiver without infinite paths. Furthermore, specialized to the $\operatorname{module}$ category $\bmod A$ of a finitedimensional algebra $A$, our criteria become surprisingly easy to verify; see (3.1). As a consequence, an Auslander-Reiten component with sections of $\bmod A$ is standard if and only if it is generalized standard and if and only if it is the connecting component of a tilted factor algebra of $A$. Finally, we point out that some of our results will be applied in the future to study cluster categories of infinite Dynkin types.

\section{Standard COMPONEnTS haVing SECTIONS}

Throughout this paper, $k$ stands for an arbitrary field. A $k$-category is a category in which the morphism sets are $k$-vector spaces and the composition of morphisms is $k$-bilinear. A $k$-category is called Hom-finite if its morphism spaces are all finitedimensional over $k$, and Krull-Schmidt if every non-zero object is a finite direct sum of objects with a local endomorphism algebra.

For the rest of this section, let $\mathcal{C}$ stand for a Hom-finite Krull-Schmidt additive $k$ category. The radical morphisms in $\mathcal{C}$ are those in the $\operatorname{Jacobson} \operatorname{radical} \operatorname{rad}(\mathcal{C})$. One calls $\operatorname{rad}^{\infty}(\mathcal{C})=\bigcap_{n \geq 1} \operatorname{rad}^{n}(\mathcal{C})$ the infinite radical of $\mathcal{C}$, where $\operatorname{rad}^{n}(\mathcal{C})$ is the $n$-th power of $\operatorname{rad}(\mathcal{C})$. Two objects $X, Y$ in $\mathcal{C}$ are said to be orthogonal if $\operatorname{Hom}_{\mathcal{C}}(X, Y)=0$ and $\operatorname{Hom}_{\mathcal{C}}(Y, X)=0$. If $X \in \mathcal{C}$ is indecomposable, then the division algebra $k_{X}=\operatorname{End}(X) / \operatorname{rad}(X, X)$ is called the automorphism field of $X$, and we shall call $X$ a brick provided that $\operatorname{End}_{\mathcal{C}}(X)$ is trivial, that is, $\operatorname{End}_{\mathcal{C}}(X) \cong k$. Let $f: X \rightarrow Y$ be a morphism in $\mathcal{C}$. One says that $f$ is irreducible if it is neither a section nor a retraction, and any factorization $f=g h$ implies that $h$ is a section or $g$ is a retraction. Moreover, $f$ is called left almost split if it is not a section and every non-section morphism $g: X \rightarrow M$ in $\mathcal{C}$ factors through $f$, and left minimal if every endomorphism $h$ of $Y$ such that $f=h f$ is an automorphism. In a dual manner, one defines $f$ to be right almost split and right minimal. Further, $f$ is called a source morphism for $X$ if it is left minimal and left almost split, and a sink morphism for $Y$ if it is right minimal and right almost split. A sequence of morphisms

$$
X \stackrel{f}{\longrightarrow} Y \stackrel{g}{\longrightarrow} Z
$$

in $\mathcal{C}$ with $Y \neq 0$ is called almost split provided that $f$ is a source morphism and a pseudo-kernel of $g$, while $g$ is a sink morphism and a pseudo-cokernel of $f$; see [11. (1.3)]. In case $\mathcal{C}$ is abelian or triangulated, the definition of an almost split sequence given here coincides somehow with the classical one; see [11, (1.5), (6.1)].

1.1. Lemma. Let $\mathcal{C}$ have an almost split sequence as follows:

$$
X \stackrel{\left(\begin{array}{l}
f_{1} \\
f_{2}
\end{array}\right)}{\longrightarrow} Y_{1} \amalg Y_{2} \stackrel{\left(g_{1}, g_{2}\right)}{\longrightarrow} Z .
$$

(1) There exists a $k$-linear isomorphism $k_{X} \cong k_{Z}$.

(2) If $u: M \rightarrow Y_{1}$ is a morphism in $\mathcal{C}$ such that $g_{1} u=0$, then there exists some $w: M \rightarrow X$ such that $u=f_{1} w$ and $f_{2} w=0$.

(3) If $v: Y_{1} \rightarrow N$ is a morphism in $\mathcal{C}$ such that $v f_{1}=0$, then there exists some $w: Z \rightarrow N$ such that $v=w g_{1}$ and $w g_{2}=0$. 
Proof. Statement (1) is implicitly stated and proved in the proof of [11, (2.1)]. Let $u: M \rightarrow Y_{1}$ be such that $g_{1} u=0$. Then $\left(g_{1}, g_{2}\right)\left(\begin{array}{l}u \\ 0\end{array}\right)=0$, and hence there exist some $w: M \rightarrow X$ such that $\left(\begin{array}{l}u \\ 0\end{array}\right)=\left(\begin{array}{l}f_{1} \\ f_{2}\end{array}\right) w$. This proves Statement (2). Dually, we can show Statement (3). The proof of the lemma is completed.

The Auslander-Reiten quiver $\Gamma_{\mathcal{C}}$ of $\mathcal{C}$ is first defined to be a valued translation quiver as follows. The vertex set is a complete set of the representatives of the isomorphism classes of the indecomposable objects in $\mathcal{C}$. For vertices $X$ and $Y$, we write $d_{X Y}^{\prime}$ and $d_{X Y}$ for the dimensions of $\operatorname{irr}(X, Y)=\operatorname{rad}(X, Y) / \operatorname{rad}^{2}(X, Y)$ over $k_{X}$ and $k_{Y}$ respectively, and draw a unique valued arrow $X \rightarrow Y$ with valuation $\left(d_{X Y}, d_{X Y}^{\prime}\right)$ if and only if $d_{X Y}>0$. The translation $\tau$ is defined so that $\tau Z=X$ if and only if $\mathcal{C}$ has an almost split sequence $X \rightarrow Y \rightarrow Z$. A valuation $\left(d_{X Y}, d_{X Y}^{\prime}\right)$ is called symmetric if $d_{X Y}=d_{X Y}^{\prime}$ and trivial if $d_{X Y}=d_{X Y}^{\prime}=1$. Next, $\Gamma_{\mathcal{C}}$ is modified in such a way that each symmetrically valued arrow $X \rightarrow Y$ is replaced by $d_{X Y}$ unvalued arrows from $X$ to $Y$. That is, $\Gamma_{\mathcal{c}}$ becomes a partially valued translation quiver in which all valuations are non-symmetric; see [11, (2.1)].

Let $\Sigma$ be a convex subquiver of $\Gamma_{\mathcal{C}}$ in which every object has a trivial automorphism field. In particular, $d_{X Y}=d_{X Y}^{\prime}$ for all $X, Y \in \Sigma$. By our construction, $\Sigma$ is a non-valued translation quiver with possible multiple arrows. Thus, one can define the path category $k \Sigma$ and the mesh category $k(\Sigma)$ of $\Sigma$ over $k$; see, for example, [13. (2.1)]. In the sequel, for $u \in k \Sigma$ we shall write $\bar{u}$ for its image in $k(\Sigma)$.

1.2. Definition. Let $\Sigma$ be a convex subquiver of $\Gamma_{\mathcal{C}}$, and let $\mathcal{C}(\Sigma)$ be the full subcategory of $\mathcal{C}$ generated by the objects in $\Sigma$. We shall say that $\Sigma$ is standard provided that every object in $\Sigma$ has a trivial automorphism field and there exists a $k$-equivalence $F: k(\Sigma) \stackrel{\sim}{\rightarrow} \mathcal{C}(\Sigma)$, which acts identically on the objects.

1.3. Lemma. Let $\Sigma$ be a convex subquiver of $\Gamma_{\mathcal{C}}$, and let $F: k(\Sigma) \stackrel{\sim}{\rightarrow} \mathcal{C}(\Sigma)$ be a $k$-equivalence acting identically on the objects. If $X, Y \in \Sigma$, then the classes $F(\bar{\alpha})+\operatorname{rad}^{2}(X, Y)$ form a $k$-basis of $\operatorname{irr}(X, Y)$, where $\alpha$ ranges over the set of arrows from $X$ to $Y$.

Proof. Let $X, Y \in \Sigma$. For $1 \leq i \leq 2$, consider the $k$-subspace $\mathcal{I}^{(i)}(X, Y)$ of $k(\Sigma)(X, Y)$ generated by $\bar{p}$, where $p$ ranges over the set of paths of length $\geq i$ from $X$ to $Y$. Write $\Sigma_{1}(X, Y)$ for the set of arrows from $X$ to $Y$. Since the mesh relations are sums of paths of length two, the classes $\bar{\alpha}+\mathcal{I}^{(2)}(X, Y)$, with $\alpha \in \Sigma_{1}(X, Y)$, are $k$-linearly independent, and hence they form a $k$-basis for $\mathcal{I}^{(1)}(X, Y) / \mathcal{I}^{(2)}(X, Y)$. Thus, $\mathcal{I}^{(1)}(X, Y) / \mathcal{I}^{(2)}(X, Y)$ and $\operatorname{irr}(X, Y)$ are of the same $k$-dimension. Since $F$ induces a $k$-isomorphism $F: k(\Sigma)(X, Y) \rightarrow \operatorname{Hom}_{\mathcal{C}}(X, Y)$, it is easy to see that $F$ induces a $k$-epimorphism $F: \mathcal{I}^{(1)}(X, Y) \rightarrow \operatorname{rad}(X, Y)$. In particular, $F$ maps $\mathcal{I}^{(2)}(X, Y)$ into $\operatorname{rad}^{2}(X, Y)$. This yields a $k$-epimorphism

$$
\bar{F}: \mathcal{I}^{(1)}(X, Y) / \mathcal{I}^{(2)}(X, Y) \rightarrow \operatorname{irr}(X, Y): u+\mathcal{I}^{(2)}(X, Y) \mapsto F(u)+\operatorname{rad}^{2}(X, Y),
$$

which is necessarily an isomorphism. The proof of the lemma is completed.

Given a quiver $\Sigma$ with no oriented cycle, one constructs a stable translation quiver $\mathbb{Z} \Sigma$; see, for example, [13, (2.1)]. We denote by $\mathbb{N} \Sigma$ the full translation subquiver of $\mathbb{Z} \Sigma$ generated by the vertices $(n, x)$ with $n \geq 0$ and $x \in \Sigma$, and by $\mathbb{N}^{-} \Sigma$ the one generated by the vertices $(n, x)$ with $n \leq 0$ and $x \in \Sigma$. Now, let $\Gamma$ be a connected component of $\Gamma_{\mathcal{C}}$. A connected full subquiver $\Delta$ of $\Gamma$ is 
called a section if it is convex in $\Gamma$, contains no oriented cycle, and meets every $\tau$-orbit in $\Gamma$ exactly once. In this case, every object in $\Gamma$ is uniquely written as $\tau^{n} X$ with $n \in \mathbb{Z}$ and $X \in \Delta$, and there exists a translation-quiver embedding $\Gamma \rightarrow \mathbb{Z} \Delta: \tau^{n} X \mapsto(-n, X)$; see [10, (2.3)]. We denote by $\Delta^{-}$the full subquiver of $\Gamma$ generated by the vertices $\tau^{n} X$ with $n>0$ and $X \in \Delta$, and by $\Delta^{+}$the one generated by the vertices $\tau^{n} X$ with $n<0$ and $X \in \Delta$. The section $\Delta$ is called right-most if $\Delta^{+}=\emptyset$ and left-most if $\Delta^{-}=\emptyset$. Observe that $\Gamma$ has at most one right-most section and at most one left-most section.

In order to state and prove the main result of this section, we need some terminology and notation. Firstly, an infinite path in a quiver is called left infinite if it has no starting point and right infinite if it has no ending point. Secondly, given two (possibly empty) subquivers $\Sigma, \Omega$ of $\Gamma_{\mathcal{C}}$, we shall write $\operatorname{Hom}_{\mathcal{C}}(\Sigma, \Omega)=0$ in case $\operatorname{Hom}_{\mathcal{C}}(X, Y)=0$ for all possible objects $X \in \Sigma$ and $Y \in \Omega$.

1.4. Theorem. Let $\mathcal{C}$ be a Hom-finite Krull-Schmidt additive $k$-category, and let $\Gamma$ be a connected component of $\Gamma_{\mathcal{C}}$ having a section $\Delta$. If $\Delta^{+}$has no left infinite path and $\Delta^{-}$has no right infinite path, then $\Gamma$ is standard if and only if $\Delta$ is standard such that $\operatorname{Hom}_{\mathcal{C}}\left(\Delta^{+}, \Delta \cup \Delta^{-}\right)=0$ and $\operatorname{Hom}_{\mathcal{C}}\left(\Delta, \Delta^{-}\right)=0$.

Proof. Suppose that $\Delta^{+}$has no left infinite path and $\Delta^{-}$has no right infinite path. Assume first that $\Gamma$ is standard. In particular, $\Delta$ is standard. Since $\Gamma$ embeds in $\mathbb{Z} \Delta$, we see that $\Gamma$ has no path from $X$ to $Y$ in case $X \in \Delta^{+}$and $Y \in \Delta \cup \Delta^{-}$, or $X \in \Delta$ and $Y \in \Delta^{-}$. This shows the necessity.

Assume conversely that $\Delta$ is standard such that $\operatorname{Hom}_{\mathcal{C}}\left(\Delta^{+}, \Delta \cup \Delta^{-}\right)=0$ and $\operatorname{Hom}_{\mathcal{C}}\left(\Delta, \Delta^{-}\right)=0$. In particular, every object in $\Delta$ has a trivial endomorphism algebra. Being of the form $\tau^{n} X$ with $n \in \mathbb{Z}$ and $X \in \Delta$, by Lemma 1.1(1), every object in $\Gamma$ has a trivial automorphism field. By definition, every object in $\Delta^{+}$is the ending term of an almost split sequence in $\mathcal{C}$. Let $X$ be an object lying in $\Delta^{+}$. Admitting a sink morphism, $X$ has only finitely many immediate predecessors in $\Gamma$, and in particular, it has at most finitely many immediate predecessors in $\Delta^{+}$. Moreover, if $Y$ is an immediate successor of $X$ in $\Delta^{+}$, then $\tau Y$ is an immediate predecessor of $X$ in $\Gamma$. Therefore, $X$ has at most finitely many immediate successors in $\Delta^{+}$. That is, $\Delta^{+}$is locally finite. Further, since $\Delta^{+}$has no left infinite path, it follows from König's Lemma that $\Delta^{+}$has only finitely many paths ending in any pre-fixed object. Thus, for each object $M \in \Delta \cup \Delta^{+}$, we may define an integer $n_{M} \geq 0$ in such a way that $n_{M}=0$ if $M \in \Delta$; otherwise, $n_{M}-1$ is the maximal length of the paths in $\Delta^{+}$ending in $M$. The following statement is evident.

(1) Let $p: X \rightsquigarrow Y$ be a non-trivial path in $\Gamma$. If $X \in \Delta \cup \Delta^{+}$, then $Y \in \Delta \cup \Delta^{+}$ with $n_{X} \leq n_{Y}$, and the equality occurs if and only if $X, Y \in \Delta$.

For each $n \geq 0$, denote by $\Gamma^{n}$ the full subquiver of $\Gamma$ generated by the vertices $X \in \Delta \cup \Delta^{+}$with $n_{X} \leq n$, which is clearly convex in $\Gamma$. Moreover, denote by $\Gamma^{+}$the union of the $\Gamma^{n}$ with $n \geq 0$, that is, the full subquiver of $\Gamma$ generated by $\Delta^{+} \cup \Delta$. The following statement is an immediate consequence of Statement (1).

(2) If $p: X \rightsquigarrow Y$ is a non-trivial path in $\Gamma^{n+1}$ with $n \geq 0$, then $X \in \Gamma^{n}$, and consequently, $p \notin \Gamma^{n}$ if and only if $Y \notin \Gamma^{n}$.

Now, let $F^{0}: k(\Delta) \stackrel{\sim}{\rightarrow} \mathcal{C}(\Delta)$ be a $k$-linear equivalence, acting identically on the objects. Since $\Delta$ contains no mesh of $\Gamma$, we have $k(\Delta)=k \Delta$. Assume that $n \geq 0$ and $F^{0}$ extends to a full $k$-linear functor $F^{n}: k \Gamma^{n} \rightarrow \mathcal{C}\left(\Gamma^{n}\right)$, acting identically 
on the objects and having a kernel generated by the mesh relations. In order to extend $F^{n}$ to $k \Gamma^{n+1}$, we shall need the following statement.

(3) If $f: X \rightarrow Y$ is a non-zero radical morphism in $\mathcal{C}\left(\Gamma^{n+1}\right)$, then $\Gamma^{n+1}$ has a non-trivial path from $X$ to $Y$. Let $f: X \rightarrow Y$ be a non-zero radical morphism in $\mathcal{C}\left(\Gamma^{n+1}\right)$. Assume on the contrary that $\Gamma^{n+1}$ has no non-trivial path from $X$ to $Y$. We then claim that $\Delta$ has an object $M$, which is a predecessor of $Y$ in $\Gamma^{n+1}$, such that $\operatorname{Hom}_{\mathcal{C}}(X, M) \neq 0$. Indeed, suppose that this claim was false. In particular, $Y \notin \Delta$, and hence $Y \in \Delta^{+}$. Since $\Delta$ is a section of $\Gamma$, every immediate predecessor of an object in $\Delta^{+}$lies in $\Delta^{+} \cup \Delta$. Since every object in $\Delta^{+}$admits a sink morphism in $\mathcal{C}$, by factorizing the radical morphism $f$ and using our assumption and the nonvalidity of our claim, we obtain a left infinite path

$$
\cdots \longrightarrow Y_{i} \longrightarrow Y_{i-1} \longrightarrow \cdots \longrightarrow Y_{1} \longrightarrow Y
$$

in $\Delta^{+}$such that $\operatorname{Hom}_{\mathcal{C}}\left(X, Y_{i}\right) \neq 0$ for all $i>0$, contrary to the hypothesis on $\Delta^{+}$. Thus, $\Delta$ has the claimed object $M$. Since $\operatorname{Hom}_{\mathcal{C}}\left(\Delta^{+}, \Delta\right)=0$, we have $X \in \Delta$. Since $k \Delta \cong \mathcal{C}(\Delta)$, there exists a path in $\Delta$ from $X$ to $M$. This yields a non-trivial path in $\Gamma^{n+1}$ from $X$ to $Y$, contrary to our assumption. Statement (3) is established.

Fix $Z \in \Gamma^{n+1} \backslash \Gamma^{n}$. Observe that $Z \in \Delta^{+}$and $\tau Z \in \Delta^{+} \cup \Delta$. Thus, $k \Gamma^{n+1}$ has a mesh relation $\delta_{Z}=\sum_{i=1}^{r} \beta_{i} \alpha_{i}$, where $\alpha_{i}: \tau Z \rightarrow Y_{i}, i=1, \ldots, r$, are the arrows starting in $\tau Z$ and $\beta_{i}: Y_{i} \rightarrow Z, i=1, \ldots, r$, are the arrows ending in $Z$. By Statement $(2), \tau Z, Y_{1}, \ldots, Y_{r} \in \Gamma^{n}$. Since $\tau Z$ admits a source morphism in $\mathcal{C}$, it follows from Lemma 1.3 that $f=\left(F^{n}\left(\alpha_{1}\right), \ldots, F^{n}\left(\alpha_{r}\right)\right)^{T}: \tau Z \rightarrow Y_{1} \oplus \cdots \oplus Y_{r}$ is a source morphism, which embeds in an almost split sequence

$$
\tau Z \stackrel{f}{\longrightarrow} Y_{1} \oplus \cdots \oplus Y_{r} \stackrel{\left(g_{1}, \ldots, g_{r}\right)}{\longrightarrow} Z
$$

in $\mathcal{C}$; see [11, (1.4)]. Set $F^{n+1}(Z)=Z, F^{n+1}\left(\varepsilon_{z}\right)=\mathbf{1}_{z}$, where $\varepsilon_{z}$ is the trivial path at $Z$, and $F^{n+1}\left(\beta_{i}\right)=g_{i}$, for $i=1, \ldots, s$. In view of Statement (2), we have defined $F^{n+1}$ on the vertices, the trivial paths, and the arrows in $\Gamma^{n+1}$. In an evident manner, we may extend $F^{n}$ to a $k$-functor $F^{n+1}: k \Gamma^{n+1} \rightarrow \mathcal{C}\left(\Gamma^{n+1}\right)$, acting identically on the objects.

Let $u: Y \rightarrow Z$ be a non-zero radical morphism in $\mathcal{C}\left(\Gamma^{n+1}\right)$. By Statement (3), $\Gamma^{n+1}$ has a non-trivial path from $Y$ to $Z$, and hence $Y \in \Gamma^{n}$ by Statement (2). If $Z \in \Gamma^{n}$ then, by the induction hypothesis, $u=F^{n}(\rho)$ for some morphism $\rho: Y \rightarrow Z$ in $k \Gamma^{n}$. Otherwise, $Z$ is the ending term of an almost split sequence $(*)$ as stated above. Then $u=\sum_{i=1}^{r} g_{i} u_{i}$, with morphisms $u_{i}: Y \rightarrow Y_{i}$ in $\mathcal{C}$. Since $Y_{i} \in \Gamma^{n}$, there exists $\rho_{i}: Y \rightarrow Y_{i}$ in $k \Gamma^{n}$ such that $u_{i}=F^{n}\left(\rho_{i}\right)$, for $i=1, \ldots, r$. This yields $u=F^{n+1}\left(\sum_{i=1}^{r} \beta_{i} \rho_{i}\right)$; that is, $F^{n+1}$ is full.

Next we shall show, for $\theta \in k \Gamma^{n+1}$, that $F^{n+1}(\theta)=0$ if and only if $\theta$ lies in the mesh ideal of $k \Gamma^{n+1}$. In view of the induction hypothesis, we may assume that $\theta$ is non-zero of the form $\theta: Y \rightarrow Z$ with $Z \in \Gamma^{n+1} \backslash \Gamma^{n}$. In particular, $\Gamma^{n+1}$ has a non-trivial path from $Y$ to $Z$. By Statement (2), $Y \in \Gamma^{n}$. Suppose first that $\theta$ lies in the mesh ideal of $k \Gamma^{n+1}$. For simplicity, we may assume that $\theta=\zeta \delta \sigma$, where $\sigma, \delta, \zeta \in k \Gamma^{n+1}$ with $\delta$ a mesh relation. If $\zeta$ has as a non-zero summand a multiple of a non-trivial path, then $\delta \in k \Gamma^{n}$ by Statement (2). Hence, $F^{n+1}(\theta)=0$ by the induction hypothesis. Otherwise, $\delta$ is the mesh relation $\delta_{Z}$ as stated above, and $\theta=\left(\sum_{i=1}^{r} \beta_{i} \alpha_{i}\right) \eta$, where $\eta: Y \rightarrow \tau Z$ is a morphism in $k \Gamma^{n}$. Since $(*)$ is an almost split sequence, we obtain $F^{n+1}(\theta)=0$. 
Suppose conversely that $F^{n+1}(\theta)=0$. Consider the mesh relation $\delta_{Z}$ and the almost split sequence $(*)$ as stated above. Then $\theta=\sum_{i=1}^{r} \beta_{i} \theta_{i}$, where $\theta_{i}: Y \rightarrow Y_{i}$ is in $k \Gamma^{n}$. Since $\sum_{i=1}^{r} F^{n+1}\left(\beta_{i}\right) F^{n}\left(\theta_{i}\right)=F^{n+1}(\theta)=0$, there exists $v: Y \rightarrow \tau Z$ in $\mathcal{C}$ such that $F^{n}\left(\theta_{i}\right)=F^{n}\left(\alpha_{i}\right) v$, for $i=1, \ldots, r$. Since $F^{n}$ is full, $v=F^{n}(\eta)$ with $\eta: Y \rightarrow \tau Z$ in $k \Gamma^{n}$. Hence $F^{n}\left(\theta_{i}\right)=F^{n}\left(\alpha_{i} \eta\right)$, and by the induction hypothesis, $\theta_{i}-\alpha_{i} \eta$ lies in the mesh ideal of $k \Gamma^{n}, i=1, \ldots, r$. As a consequence,

$$
\theta=\sum_{i=1}^{r} \beta_{i}\left(\theta_{i}-\alpha_{i} \eta\right)+\left(\sum_{i=1}^{r} \beta_{i} \alpha_{i}\right) \eta
$$

lies in the mesh ideal of $k \Gamma^{n+1}$. This shows that $F^{n+1}$ is full and its kernel is generated by the mesh relations. By induction, $F^{0}$ extends to a full $k$-functor $F^{+}: k \Gamma^{+} \rightarrow \mathcal{C}\left(\Gamma^{+}\right)$, acting identically on the objects and having a kernel generated by the mesh relations.

Finally, for each object $N \in \Gamma$, we may define $m_{N} \geq 0$ so that $m_{N}=0$ if $N \in \Gamma^{+}$; otherwise, $m_{N}-1$ is the maximal length of the paths in $\Delta^{-}$which start in $N$. For $m \geq 0$, denote by $\Gamma^{(m)}$ the full subquiver of $\Gamma$ generated by the objects $Y$ with $m_{Y} \leq m$. Then $\Gamma$ is the union of $\Gamma^{(m)}$ with $m \geq 0$. In a dual manner, we may apply the induction on $m$ to show that $F^{+}$extends to a full $k$-functor $F: k \Gamma \rightarrow \mathcal{C}(\Gamma)$, which acts identically on the objects and has a kernel generated by the mesh relations. The proof of the theorem is completed.

The following result is useful for verifying the conditions stated in Theorem 1.4

1.5. Lemma. Let $\Gamma$ be a connected component of $\Gamma_{\mathcal{c}}$, containing a section $\Delta$.

(1) If $\Delta$ has no left infinite path, then $\Delta^{+}$has no left infinite path.

(2) If $\Delta$ has no right infinite path, then $\Delta^{-}$has no right infinite path.

Proof. It suffices to prove Statement (1). Suppose that $\Delta^{+}$has a left infinite path

$$
\cdots \rightarrow \tau^{-n_{i}} X_{i} \rightarrow \cdots \rightarrow \tau^{-n_{1}} X_{1} \rightarrow \tau^{-n_{0}} X_{0},
$$

where $X_{i} \in \Delta$ and $n_{i}>0$. Since $\Gamma$ embeds in $\mathbb{Z} \Delta$ (see [10, (2.3)]), we see that $n_{i} \leq n_{i-1}$ for all $i>0$. As a consequence, there exists $r \geq 0$ such that $n_{i}=n_{r}$ for $i \geq r$. This yields a left infinite path

$$
\cdots \longrightarrow X_{i} \longrightarrow \cdots \longrightarrow X_{r}
$$

in $\Delta$. The proof of the lemma is completed.

We shall say that a sink morphism in $\mathcal{C}$ is proper if it either is a monomorphism or fits in an almost slit sequence; dually, a source morphism is proper if it either is an epimorphism or fits in an almost slit sequence. Observe that sink or source morphisms in an abelian category are all proper. The following result is a generalization of Lemma 3 stated in [13, (2.3)].

1.6. Theorem. Let $\mathcal{C}$ be a Hom-finite Krull-Schmidt additive $k$-category. Let $\Gamma$ be a connected component of $\Gamma_{\mathcal{C}}$, and let $\Delta$ be a section of $\Gamma$ in which every object has a trivial automorphism field and admits a proper sink morphism as well as a proper source morphism. If $\Delta$ has no infinite path, then $\Gamma$ is standard if and only if $\operatorname{Hom}_{\mathcal{C}}\left(\Delta^{+}, \Delta^{-}\right)=0$.

Proof. Suppose that $\Delta$ has no infinite path. By Lemma 1.5 $\Delta^{+}$has no left infinite path and $\Delta^{-}$has no right infinite path. We shall need the following statement. 
Sub-lemma. Let $M \in \Gamma$ with $\operatorname{Hom}_{\mathcal{C}}\left(M, \Delta^{-}\right)=0$, and let $N \in \Delta$. If $\mathcal{C}$ has a non-zero radical morphism $f: M \rightarrow N$, then $\Gamma$ has a non-trivial path $M \rightsquigarrow N$.

Indeed, suppose that $\Gamma$ has no non-trivial path from $M$ to $N$. By assumption, $N$ admits a sink morphism $g=\left(g_{1}, \cdots, g_{r}\right): N_{1} \oplus \cdots \oplus N_{r} \rightarrow N$, where $N_{i} \in \Gamma$. If $f: M \rightarrow N$ is non-zero and radical, then $f=\sum_{i=1}^{r} g_{i} f_{i}$, with $f_{i}: M \rightarrow N_{i}$ in $\mathcal{C}$. We may assume that $f_{1}$ is non-zero. Since $\Delta$ is a section, $N_{1} \in \Delta \cup \tau \Delta$; see [10, (2.2)]. Since $\operatorname{Hom}_{\mathcal{C}}\left(M, \Delta^{-}\right)=0$, we have $N_{1} \in \Delta$. Since $\Gamma$ has no path from $M$ to $N_{1}$, we see that $f_{1}$ is radical. Repeating this process, we see that $\Delta$ contains an infinite path ending in $N$, a contradiction. This proves the sub-lemma.

Now, assume that $\operatorname{Hom}_{\mathcal{C}}\left(\Delta^{+}, \Delta^{-}\right)=0$. We deduce from the above sub-lemma that $\operatorname{Hom}_{\mathcal{C}}\left(\Delta^{+}, \Delta\right)=0$. Using the dual statement, we obtain $\operatorname{Hom}_{\mathcal{C}}\left(\Delta, \Delta^{-}\right)=0$. It remains to construct a $k$-linear equivalence $F: k \Delta \rightarrow \mathcal{C}(\Delta)$. Since every object in $\Delta$ has a trivial automorphism field, so do the objects in $\Gamma$. Set $F(X)=X$ and $F\left(\varepsilon_{X}\right)=\mathbf{1}_{X}$ for $X \in \Delta$. Let $X, Y \in \Delta$ with $d=d_{X Y}>0$. If $\alpha_{i}: X \rightarrow Y$, $i=1, \ldots, d$, are the arrows from $X$ to $Y$, then we choose irreducible morphisms $f_{\alpha_{i}}: X \rightarrow Y$ such that $f_{\alpha_{1}}+\operatorname{rad}^{2}(X, Y), \ldots, f_{\alpha_{r}}+\operatorname{rad}^{2}(X, Y)$ form a $k$-basis of $\operatorname{irr}(X, Y)$, and set $F\left(\alpha_{i}\right)=f_{\alpha_{i}}, i=1, \ldots, d$. In an evident manner, we obtain a $k$-linear functor $F: k \Delta \rightarrow \mathcal{C}(\Delta)$.

We claim that $F$ induces a $k$-isomorphism $F_{X Y}: \operatorname{Hom}_{k \Delta}(X, Y) \rightarrow \operatorname{Hom}_{\mathcal{C}}(X, Y)$, for any $X, Y \in \Delta$. Since every object in $\Delta$ admits a sink morphism and a source morphism, $\Delta$ is locally finite. Having no infinite path, by König's Lemma, $\Delta$ has at most finitely many paths from $X$ to $Y$. Define an integer $n_{X Y}$ in such a way that $n_{X Y}=-1$ if $\Delta$ has no path from $X$ to $Y$; otherwise, $n_{X Y}$ is the maximal length of the paths from $X$ to $Y$. If $n_{X Y}=-1$, then the claim follows easily from the above statement. If $n_{X Y}=0$, then $\operatorname{Hom}_{k \Delta}(X, Y)=k \varepsilon_{Y}$. On the other hand, $\operatorname{Hom}_{\mathcal{C}}(X, Y)=k \mathbf{1}_{Y}$ by the above sub-lemma, and the claim follows.

Suppose that $n_{X Y}>0$. Let $\beta_{i}: Z_{i} \rightarrow Y, i=1, \ldots, s$, be the arrows in $\Delta$ ending in $Y$. Then $n_{X Z_{i}}<n_{X Y}$, and $\left(f_{\beta_{1}}, \cdots, f_{\beta_{s}}\right): Z_{1} \oplus \cdots \oplus Z_{s} \rightarrow Y$ is irreducible; see [2, (3.4)]. Since $Y$ admits a proper sink morphism, there exists a morphism $u: U \rightarrow Y$ such that $v=\left(f_{\beta_{1}}, \cdots, f_{\beta_{s}}, u\right): Z_{1} \oplus \cdots \oplus Z_{s} \oplus U \rightarrow Y$ is a proper sink morphism. Let $h: X \rightarrow Y$ be a morphism in $\mathcal{C}$. Being radical, $h$ factors through $v$. Since $\Delta$ is a section, every indecomposable summand of $U$ lies in $\tau \Delta$, and since $\operatorname{Hom}_{\mathcal{C}}\left(X, \Delta^{-}\right)=0$, we have $h=f_{\beta_{1}} h_{1}+\cdots+f_{\beta_{s}} h_{s}$, with morphisms $h_{i}: X \rightarrow Z_{i}$ in $\mathcal{C}$. For each $1 \leq i \leq s$, by the induction hypothesis, $h_{i}$ is a sum of composites of the chosen irreducible morphisms. Therefore, $h$ is a sum of composites of the chosen irreducible morphisms. Hence, $F_{X Y}$ is surjective. Next, let $\rho: X \rightarrow Y$ be in $k \Delta$ such that $F(\rho)=0$. Then $\rho=\beta_{1} \rho_{1}+\cdots+\beta_{s} \rho_{s}$, where the $\rho_{i}: X \rightarrow Z_{i}$ are in $k \Delta$. Set $w=\left(F\left(\rho_{1}\right), \cdots, F\left(\rho_{s}\right)\right)^{T}: X \rightarrow Z_{1} \oplus \cdots \oplus Z_{s}$. Then $\left(f_{\beta_{1}}, \cdots, f_{\beta_{s}}\right) w=F(\rho)=0$. If $\mathcal{C}$ has an almost split sequence ending in $Y$, then, by Lemma 1.1 (2), $w$ factors through $\tau Y$, and since $\operatorname{Hom}_{\mathcal{C}}\left(X, \Delta^{-}\right)=0$, we have $w=0$. Otherwise, $v$ is a monomorphism, and hence, $w=0$. That is, in any case, $F\left(\rho_{i}\right)=0$, and by the inductive hypothesis, $\rho_{i}=0, i=1, \ldots, s$. As a consequence, $\rho=0$. Thus, $F_{X Y}$ is injective. This implies that $F$ is an equivalence. By Theorem 1.4 $\Gamma$ is standard. This establishes the sufficiency, and the necessity is evident. The proof of the theorem is completed.

Let $\Sigma$ be a convex subquiver of $\Gamma_{\mathcal{C}}$. We shall say that $\Sigma$ is schurian if, for any objects $X, Y$ in $\Sigma$, the $k$-space $\operatorname{Hom}_{\mathcal{C}}(X, Y)$ is of dimension at most one, and it vanishes whenever $Y$ is a not successor of $X$ in $\Sigma$. Moreover, we call $\Sigma$ a wing of 
rank $n$ if it is trivially valued of the following shape:

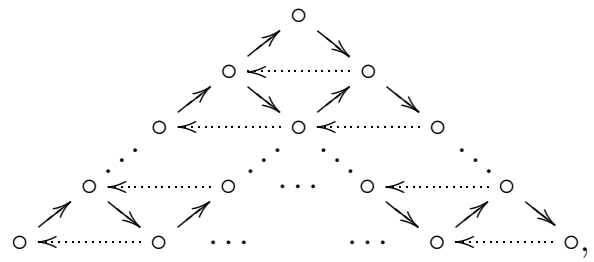

where the dotted arrows indicate the action of $\tau$, the objects are pairwise distinct and the number of $\tau$-orbits is $n$; see [13, (3.3)]. In this case, the object on the top is called the wing vertex, and the objects at the bottom are said to be quasi-simple.

1.7. Lemma. Let $\mathcal{W}$ be a wing of $\Gamma_{\mathcal{c}}$. If the quasi-simple objects in $\mathcal{W}$ are pairwise orthogonal bricks, then $\mathcal{W}$ is schurian.

Proof. Assume that the quasi-simple objects in $\mathcal{W}$ are pairwise orthogonal bricks. Let $n$ be the rank of $\mathcal{W}$. If $n=1$, then the lemma holds trivially. Suppose that $n>1$ and the lemma holds for wings of rank $n-1$. Write the objects in $\mathcal{W}$ as $X_{i j}$ with $1 \leq i \leq n$ and $1 \leq j \leq i$ so that $X_{11}$ is the wing vertex, the $X_{n j}$ with $1 \leq j \leq n$ are the quasi-simple objects, and $\tau X_{i j}=X_{i, j+1}$ for $1<i \leq n$ and $1 \leq j<i$. Observe that $X_{21}$ is the wing vertex of a schurian wing $\mathcal{W}_{1}$, while $X_{22}$ is the wing vertex of a schurian wing $\mathcal{W}_{2}$. It is evident that we may choose irreducible morphisms $f_{i j}: X_{i j} \rightarrow X_{i+1, j}$ for $j \leq i<n$ and $1 \leq j<n$ and irreducible morphisms $g_{p q}: X_{p q} \rightarrow X_{p-1, q-1}$ for $q \leq p \leq n$ and $2 \leq q \leq n$ such that

$$
\mathcal{E}\left(X_{n j}\right): X_{n, j+1} \stackrel{g_{n, j+1}}{\longrightarrow} X_{n-1, j} \stackrel{f_{n-1, j}}{\longrightarrow} X_{n j}
$$

is an almost split sequence for $j=1, \ldots, n-1$, and

$$
\mathcal{E}\left(X_{i j}\right): \quad X_{i, j+1} \stackrel{\left(g_{i, j+1}, f_{i, j+1}\right)}{\longrightarrow} X_{i-1, j} \oplus X_{i+1, j+1} \stackrel{\left(\begin{array}{c}
f_{i-1, j} \\
g_{i+1, j+1}
\end{array}\right)}{\longrightarrow} X_{i j}
$$

is an almost split sequence for $1 \leq j<n$ and $j<i<n$. Next, we shall divide the proof into several sub-lemmas.

(1) $\operatorname{Hom}_{\mathcal{C}}\left(X_{n 1}, X_{i i}\right)=0$ and $\operatorname{Hom}_{\mathcal{C}}\left(X_{i 1}, X_{n n}\right)=0$, for $1 \leq i \leq n$. Suppose that $\mathcal{C}$ has a non-zero morphism $f: X_{n 1} \rightarrow X_{r r}$ for some $1 \leq r \leq n$. Assume that $r$ is maximal. Since $X_{n 1}, X_{n n}$ are orthogonal, we have $r<n$. Since $\mathcal{W}_{1}$ is schurian, $f_{r r} f=0$. Applying Lemma 1.1 (2) to the almost split sequence $\mathcal{E}\left(X_{r+1, r}\right)$, we see that $f$ factors through $g_{r+1, r+1}: X_{r+1, r+1} \rightarrow X_{r r}$, which contradicts the maximality of $r$. The first part of the statement is established. In a dual manner, we may prove the second part.

(2) $\operatorname{Hom}_{\mathcal{C}}\left(X_{i 1}, \mathcal{W}_{2}\right)=0$ and $\operatorname{Hom}_{\mathcal{C}}\left(\mathcal{W}_{1}, X_{i i}\right)=0$, for $1 \leq i \leq n$. Suppose that $f: X_{s 1} \rightarrow X$ is a non-zero morphism with $1 \leq s \leq n$ and $X \in \mathcal{W}_{2}$, which is necessarily radical. If $X \neq X_{j j}$ for any $2 \leq j \leq n$, then $X$ admits a sink morphism whose domain is a direct sum of one or two objects in $\mathcal{W}_{2}$. Factorizing $f$ through this sink morphism, we obtain a non-zero morphism $g: X_{s 1} \rightarrow X_{i i}$ with $2 \leq i \leq n$. Assume that $s$ is maximal for this property. By Statement (1), $s<n$. Since $\mathcal{W}_{2}$ is schurian, $g g_{s+1,2}=0$. Applying Lemma 1.1(3) to $\mathcal{E}\left(X_{s+1,1}\right)$, we see that $g$ factors through $f_{s 1}: X_{s 1} \rightarrow X_{s+1,1}$, which contradicts the maximality of $s$. The first part of the statement is established. In a dual fashion, we may establish the second part. 
(3) $\operatorname{Hom}_{\mathcal{C}}\left(X_{n n}, \mathcal{W}_{1}\right)=0$ and $\operatorname{Hom}_{\mathcal{C}}\left(\mathcal{W}_{2}, X_{n 1}\right)=0$. Suppose that $\mathcal{C}$ has a nonzero morphism $f: X_{n n} \rightarrow X_{p q}$ with $2 \leq p \leq n$ and $1 \leq q<p$. We may assume that $p$ is maximal for this property. Since the quasi-simple objects are orthogonal, $p<n$. By the maximality of $p$, we have $f_{p q} f=0$. Applying Lemma 1.1(2) to $\mathcal{E}\left(X_{p+1, q}\right)$, we see that $f$ factors through $g_{p+1, q+1}$, contrary to the maximality of $p$. The first part of the statement is established, and the second part follows dually.

(4) If $f: X_{i i} \rightarrow X_{11}$ with $1 \leq i<n$ is such that $f g_{i+1, i+1} \cdots g_{n n}=0$, then $f=0$. Dually, if $g: X_{11} \rightarrow X_{i 1}$ is a morphism with $1 \leq i<n$ such that $f_{n-1,1} \cdots f_{i 1} g=0$, then $g=0$. Suppose that $f g_{i+1, i+1} \cdots g_{n n}=0$, but $f \neq 0$. Let $r$ with $i+1 \leq r \leq n$ be minimal such that $f g_{i+1, i+1} \cdots g_{r r}=0$. Write $f g_{i+1, i+1} \cdots g_{r r}=g g_{r r}$, where $g: X_{r-1, r-1} \rightarrow X_{11}$ is a non-zero morphism. Applying Lemma 1.1. (3) to $\mathcal{E}\left(X_{r, r-1}\right)$, we see that $g$ factors through $f_{r-1, r-1}$, which contradicts Statement (2). This establishes the first part of the statement.

(5) $\operatorname{Hom}_{\mathcal{C}}\left(X_{i i}, X_{11}\right)$ and $\operatorname{Hom}_{\mathcal{C}}\left(X_{11}, X_{i 1}\right)$ are one-dimensional, for $1 \leq i \leq n$. It suffices to prove the first part of the statement, since the second part follows dually. Let $f: X_{n n} \rightarrow X_{11}$ be a morphism. By Statement (3), $f_{11} f=0$. Applying Lemma 1.1(2) to $\mathcal{E}\left(X_{21}\right)$, we obtain some $f_{1}: X_{n n} \rightarrow X_{22}$ such that $f=g_{22} f_{1}$. Since $f_{22} f_{1}=0$ by Statement (3), we may repeat this process to obtain a morphism $f_{n-1}: X_{n n} \rightarrow X_{n n}$ such that $f=g_{22} \cdots g_{n n} f_{n-1}$. Since $\mathcal{W}_{2}$ is schurian, $f_{n-1}=$ $\mathbf{1}_{x_{n n}}$ for some $\lambda \in k$, and hence, $f=\lambda g_{22} \cdots g_{n n}$. Since $g_{22} \cdots g_{n n} \neq 0$, we see that $\left\{g_{22} \cdots g_{n n}\right\}$ is a $k$-basis for $\operatorname{Hom}_{\mathcal{C}}\left(X_{n n}, X_{11}\right)$. Write $g_{11}=\mathbf{1}_{X_{11}}$. If $g: X_{i i} \rightarrow X_{11}$ is a morphism with $1 \leq i<n$, then $g g_{i+1, i+1} \cdots g_{n n}=\mu g_{22} \cdots g_{n n}=\mu g_{11} \cdots g_{n n}$, for some $\mu \in k$. This yields that $\left(g-\mu g_{11} \cdots g_{i i}\right) g_{i+1, i+1} \cdots g_{n n}=0$. By the first part of Statement (4), $g=\mu g_{11} \cdots g_{i i}$. Being non-zero, $g_{11} \cdots g_{i i}$ forms a $k$-basis for $\operatorname{Hom}_{\mathcal{C}}\left(X_{i i}, X_{11}\right)$.

Now, suppose that $\operatorname{Hom}_{\mathcal{C}}(X, Y) \neq 0$ for some $X, Y \in \mathcal{W}$. We claim that $Y$ is a successor of $X$ and $\operatorname{Hom}_{\mathcal{C}}(X, Y)$ is one-dimensional. If $X \in \mathcal{W}_{1}$, then $Y \in \mathcal{W}_{1}$ by Statement (2). Since $\mathcal{W}_{1}$ is schurian, our claim follows. Otherwise, $X=X_{s s}$ for some $1 \leq s \leq n$. If $s=n$, then, by Statement (3), $Y=X_{i i}$ for some $1 \leq i \leq n$. Combining Statement (5) and the fact that $\mathcal{W}_{2}$ is schurian, we see that $\operatorname{Hom}_{\mathcal{C}}(X, Y)$ is one-dimensional. If $s=1$ then, by Statement (2), $Y=X_{j 1}$ for some $1 \leq j \leq n$, and hence, $\operatorname{Hom}_{\mathcal{C}}(X, Y)$ is one-dimensional by Statement (5). Finally, suppose that $1<s<n$. If $Y \in \mathcal{W}_{2}$, then our claim follows, since $\mathcal{W}_{2}$ is schurian. Otherwise, by Statement $(3), Y=X_{t 1}$ for some $1 \leq t<n$. If $t=1$, then $\operatorname{Hom}_{\mathcal{C}}(X, Y)$ is one-dimensional by Statement (5).

It remains to consider the case where $1<t<n$. Let $f: X_{s s} \rightarrow X_{t 1}$ be a nonzero morphism with $1<s, t<n$. Factorizing $f$ along the $\mathcal{E}\left(X_{j 1}\right)$ with $2 \leq j \leq t$, we get $g: X_{s s} \rightarrow X_{t+1,2}$ and $h: X_{s s} \rightarrow X_{11}$ such that $f=g_{t+1,2} g+f_{t-1,1} \cdots f_{11} h$. By Statement (5), $h=\lambda g_{22} \cdots g_{s s}$ with $\lambda \in k$. This yields $f=g_{t+1,2} u$, where $u: X_{s s} \rightarrow X_{t+1,2}$ is a non-zero morphism. Since $\mathcal{W}_{2}$ is schurian, $X_{t+1,2}$ is a successor of $X_{s s}$ and $\operatorname{Hom}_{\mathcal{C}}\left(X_{s s}, X_{t+1,2}\right)$ has a $k$-basis $\{v\}$. Therefore, $f=\mu g_{t+1,2} v$ with $\mu \in k$. This shows that $\left\{g_{t+1,2} v\right\}$ is a $k$-basis for $\operatorname{Hom}_{\mathcal{C}}\left(X_{s s}, X_{t 1}\right)$. This establishes our claim. The proof of the lemma is completed.

Let $\mathbb{A}_{\infty}^{+}$and $\mathbb{A}_{\infty}^{-}$denote the linearly oriented quivers of type $\mathbb{A}_{\infty}$ having a unique source and having a unique sink, respectively. If $\Gamma$ is a connected component of $\Gamma_{\mathcal{C}}$ of shape $\mathbb{Z} \mathbb{A}_{\infty}, \mathbb{N A}_{\infty}^{+}$or $\mathbb{N}^{-} \mathbb{A}_{\infty}^{-}$, then the objects in $\Gamma$ having at most one immediate predecessor and at most one immediate successor are called quasi-simple. 
1.8. Theorem. Let $\mathcal{C}$ be a Hom-finite Krull-Schmidt additive $k$-category, and let $\Gamma$ be a connected component of $\Gamma_{\mathcal{c}}$. If $\Gamma$ is a wing or of shape $\mathbb{Z} \mathbb{A}_{\infty}, \mathbb{N A}_{\infty}^{+}$or $\mathbb{N}^{-} \mathbb{A}_{\infty}^{-}$, then it is standard if and only if its quasi-simple objects are pairwise orthogonal bricks.

Proof. We shall need only to prove the sufficiency, since the necessity is trivial. Let $\Gamma$ be a wing or of shape $\mathbb{Z} \mathbb{A}_{\infty}, \mathbb{N A}_{\infty}^{+}$or $\mathbb{N}^{-} \mathbb{A}_{\infty}^{-}$with the quasi-simple objects being pairwise orthogonal bricks. Then any two objects in $\Gamma$ lie in a wing whose quasisimples are pairwise orthogonal bricks. By Lemma 1.7, $\Gamma$ is schurian. Choose a section $\Delta$ of $\Gamma$ so that $\Delta$ is the right-most section if $\Gamma$ is a wing or of shape $\mathbb{N}^{-} \mathbb{A}_{\infty}^{-}, \Delta$ is the left-most section if $\Gamma$ is of shape $\mathbb{N A}_{\infty}^{+}$, and $\Delta$ is any section with an alternating orientation if $\Gamma$ is of shape $\mathbb{Z} \mathbb{A}_{\infty}$. Then $\Delta^{-}$has no right infinite path and $\Delta^{+}$has no left infinite path such that $\operatorname{Hom}_{\mathcal{C}}\left(\Delta^{+}, \Delta \cup \Delta^{-}\right)=0$ and $\operatorname{Hom}_{\mathcal{C}}\left(\Delta, \Delta^{-}\right)=0$.

For each arrow $\alpha: X \rightarrow Y$ in $\Delta$, choose an irreducible morphism $f_{\alpha}: X \rightarrow Y$ in $\mathcal{C}$. Since every path in $\Delta$ is sectional, the composite of any chain of the chosen irreducible morphisms is non-zero; see [11, (2.7)]. Therefore, for any $M, N \in \Delta$, $\operatorname{Hom}_{\mathcal{C}}(M, N)$ is one-dimensional if and only if $N$ is a successor of $M$ in $\Delta$, and in this case, the composite of the chain of the chosen irreducible morphisms corresponding to the path from $M$ to $N$ forms a $k$-basis for $\operatorname{Hom}_{\mathcal{C}}(X, Y)$. It is easy to see that $k \Delta \cong$ $\mathcal{C}(\Delta)$. By Theorem 1.4 $\Gamma$ is standard. The proof of the theorem is completed.

\section{Specialization to RePresentation CATEgories of QUiVERS}

Throughout this section, we fix a connected quiver $Q=\left(Q_{0}, Q_{1}\right)$, where $Q_{0}$ is the set of vertices and $Q_{1}$ is the set of arrows, which is assumed to be strongly locally finite; that is, $Q$ is locally finite such that the number of paths between any two given vertices is finite. A $k$-representation $M$ of $Q$ consists of a family of $k$-spaces $M(x)$ with $x \in Q_{0}$, and a family of $k$-maps $M(\alpha): M(x) \rightarrow M(y)$ with $\alpha: x \rightarrow y \in Q_{1}$. For such a representation $M$, one defines its support $\operatorname{supp} M$ to be the full subquiver of $Q$ generated by the vertices $x$ for which $M(x) \neq 0$, and one calls $M$ locally finite-dimensional if $\operatorname{dim}_{k} M(x)$ is finite for all $x \in Q_{0}$, and finite-dimensional if $\Sigma_{x \in Q_{0}} \operatorname{dim}_{k} M(x)$ is finite. The locally finite-dimensional $k$ representations of $Q$ form a hereditary abelian $k$-category $\operatorname{rep}(Q)$. The subcategory of $\operatorname{rep}(Q)$ of finite-dimensional representations is written as $\operatorname{rep}^{b}(Q)$.

For each $x \in Q_{0}$, one constructs an indecomposable projective representation $P_{x}$ and an indecomposable injective representation $I_{x}$; see [3, Section 1]. Since $Q$ is strongly locally finite, $P_{x}$ and $I_{x}$ lie in $\operatorname{rep}(Q)$. One says that $M \in \operatorname{rep}(Q)$ is finitely presented if $M$ has a minimal projective presentation $P_{1} \rightarrow P_{0} \rightarrow M \rightarrow 0$, where $P_{1}, P_{0}$ are finite direct sums of some $P_{x}$ with $x \in Q_{0}$, and finitely co-presented if $M$ has a minimal injective co-presentation $0 \rightarrow M \rightarrow I_{0} \rightarrow I_{1}$, where $I_{0}, I_{1}$ are finite direct sums of some $I_{x}$ with $x \in Q_{0}$. Let $\operatorname{rep}^{+}(Q)$ and $\operatorname{rep}^{-}(Q)$ be the full subcategories of $\operatorname{rep}(Q)$ generated by the finitely presented representations and by the finitely co-presented representations, respectively. Then $\operatorname{rep}^{b}(Q)$ is the intersection of $\operatorname{rep}^{+}(Q)$ and $\operatorname{rep}^{-}(Q)$; see [3, (1.15)]. In particular, $I_{x} \in \operatorname{rep}^{+}(Q)$ if and only if $I_{x} \in \operatorname{rep}^{b}(Q)$. We shall denote by $Q^{+}$the full subquiver of $Q$ generated by the vertices $x$ for which $I_{x} \in \operatorname{rep}^{b}(Q)$. 
It is known that $\operatorname{rep}^{+}(Q)$ and $\operatorname{rep}^{-}(Q)$ are hereditary, abelian and Hom-finite; see [3. (1.15)]. In particular, they are Krull-Schmidt. The shapes of their AuslanderReiten components have been well described. Indeed, the Auslander-Reiten quiver $\Gamma_{\text {rep }^{+}(Q)}$ of $\operatorname{rep}^{+}(Q)$ has a unique preprojective component, which has a left-most section generated by the $P_{x}$ with $x \in Q_{0}$; see [3, (4.6)] and [13, (2.4)]. The connected components of $\Gamma_{\text {rep }^{+}(Q)}$ containing some of the $I_{x}$ with $x \in Q^{+}$are called preinjective and correspond bijectively to the connected components of the quiver $Q^{+}$. Note that every preinjective component has a unique right-most section generated by its injective representations $I_{x}$; see [13, (2.4)] and [3, (4.7)]. The other connected components of $\Gamma_{\mathrm{rep}^{+}(Q)}$ are called regular, and they are wings, stable tubes or of shapes $\mathbb{Z} \mathbb{A}_{\infty}, \mathbb{N A}_{\infty}^{+}$and $\mathbb{N}^{-} \mathbb{A}_{\infty}^{-}$; see [3, (4.14)], [12] and [13].

The following easy fact is well known in the finite case.

2.1. Lemma. Let $X$ and $Y$ be representations lying in $\Gamma_{\text {rep }^{+}(Q)}$. If $\tau X$ and $\tau Y$ are defined in $\Gamma_{\mathrm{rep}^{+}(Q)}$, then $\operatorname{Hom}_{\mathrm{rep}^{+}(Q)}(X, Y) \cong \operatorname{Hom}_{\mathrm{rep}^{+}(Q)}(\tau X, \tau Y)$.

Proof. Assume that $\tau X$ and $\tau Y$ are defined in $\Gamma_{\text {rep }}+(Q)$. In view of the proof stated in [3, (2.8)], we have $\operatorname{Hom}(\tau X, \tau Y) \cong D \operatorname{Ext}^{1}(Y, \tau X)$. Dually, since $\tau X$ is not injective and finite-dimensional (see [3, (3.6)]), $\operatorname{Hom}(X, Y) \cong \operatorname{DExt}^{1}(Y, \tau X)$. The proof of the lemma is completed.

Recall that $Q$ is of infinite Dynkin type if its underlying graph is $\mathbb{A}_{\infty}, \mathbb{A}_{\infty}^{\infty}$ or $\mathbb{D}_{\infty}$. In this case, a reduced walk is called a string if it contains at most finitely many, but at least one, sinks or sources. To each string $w$, one associates a string representation $M_{w}$ defined as follows: for $x \in Q_{0}$, one sets $M_{w}(x)=k$ if $x$ appears in $w$, and otherwise, $M_{w}(x)=0$; and for $\alpha \in Q_{1}$, one sets $M_{w}(\alpha)=\mathbf{1}$ if $\alpha$ appears in $w$, and otherwise, $M_{w}(\alpha)=0$; see [3, Section 5]. It is easy to see that every string representation has a trivial endomorphism algebra.

2.2. Theorem. Let $Q$ be a connected quiver which is strongly locally finite.

(1) The preprojective component and the preinjective components of $\Gamma_{\mathrm{rep}^{+}(Q)}$ are standard.

(2) If $Q$ is of finite or infinite Dynkin type, then every connected component of $\Gamma_{\text {rep }^{+}(Q)}$ is standard.

(3) If $Q$ is infinite but not of infinite Dynkin type, then $\Gamma_{\mathrm{rep}^{+}(Q)}$ has infinitely many non-standard regular components.

Proof. (1) The preprojective component $\mathcal{P}_{Q}$ of $\Gamma_{\text {rep }}{ }_{(Q)}$ has a unique left-most section $\Delta$ which is generated by $P_{x}$ with $x \in Q_{0}$ and isomorphic to $Q^{\text {op; }}$ see [3. (4.6)] and [13, (2.4)]. Hence, $\Delta^{-}=\emptyset$. Moreover, $\Delta^{+}$has no left infinite path; see [3. (4.8)]. If $f: X \rightarrow Y$ is a non-zero morphism with $X \in \mathcal{P}_{Q}$ and $Y \in \Delta$, then $X$ is a predecessor of $Y$ in $\mathcal{P}_{Q}$ (see [3. (4.9)]), and hence, $X \in \Delta$. Therefore, $\operatorname{Hom}_{\text {rep }^{+}(Q)}\left(\Delta^{+}, \Delta\right)=0$. Let $\mathcal{P}(Q)$ be the full subcategory of $\operatorname{rep}^{+}(Q)$ generated by $P_{x}$ with $x \in Q_{0}$. For each arrow $\alpha: y \rightarrow x$ in $Q$, denote by $P_{\alpha}: P_{x} \rightarrow P_{y}$ the morphism given by the right multiplication by $\alpha$. It is easy to see that

$$
F: k Q^{\mathrm{op}} \rightarrow \mathcal{P}(Q): x \mapsto P_{x} ; \alpha^{\mathrm{o}} \mapsto P_{\alpha}
$$

is a faithful $k$-functor, which is also full by Proposition 1.3 stated in [3]. Thus, $\Delta$ is standard. By Theorem 1.4 $\mathcal{P}_{Q}$ is standard. Dually, the preinjective component $\mathcal{I}_{Q}$ of $\Gamma_{\mathrm{rep}^{-}(Q)}$ is standard. Now, the preinjective components of $\Gamma_{\mathrm{rep}}{ }^{(}(Q)$ are 
precisely the connected components of the full subquiver of $\mathcal{I}_{Q}$ generated by the finite-dimensional representations; see the remark following Theorem 4.7 in 3 . On the other hand, by the dual of Lemma 4.5(1) stated in [3], the possible infinitedimensional representations lying in $\mathcal{I}_{Q}$ form a left-most section. Therefore, the possible preinjective components of $\Gamma_{\text {rep }^{+}(Q)}$ are convex translation subquivers of $\mathcal{I}_{Q}$, and in particular, they are standard.

(2) Suppose that $Q$ is of infinite Dynkin type. Let $\Gamma$ be a regular component of $\Gamma_{\text {rep }}{ }_{(Q)}$. Then $\Gamma$ is a wing or of shape $\mathbb{Z} A_{\infty}, \mathbb{N}^{-} \mathbb{A}_{\infty}^{-}$or $\mathbb{N}_{\infty}^{+}$; see [3. (4.14)]. Moreover, $Q$ is of type $\mathbb{A}_{\infty}^{\infty}$ or $\mathbb{D}_{\infty}$; see [3, (5.16)]. Assume first that $Q$ is of type $\mathbb{A}_{\infty}^{\infty}$. By Proposition 5.9 stated in [3], the representations in $\Gamma_{\text {rep }}{ }^{+}(Q)$ are all string representations, and hence, they are all bricks. Moreover, the quasi-simple representations in $\Gamma$ have pairwise disjoint supports; see [3, (5.15)]. In particular, they are pairwise orthogonal. By Theorem 1.8, $\Gamma$ is standard.

Assume next that $Q$ is of type $\mathbb{D}_{\infty}$. Then $\Gamma$ is of shape $\mathbb{Z} \mathbb{A}_{\infty}, \mathbb{N A}_{\infty}^{+}$or $\mathbb{N}^{-} \mathbb{A}_{\infty}^{-}$; see [3, (5.22)]. In particular, $\tau$ or $\tau^{-}$is defined everywhere in $\Gamma$. We shall consider only the first case, since the second case can be treated in a dual manner. Let $a \in Q_{0}$ be one of the two vertices of degree one, which lies in the support of at most two quasi-simple representations; see [3, (5.20)]. Thus, there exists a quasi-simple representation $S \in \Gamma$ such that $\left(\tau^{n} S\right)(a)=0$, for all $n \geq 0$.

Let $M, N$ be quasi-simple representations in $\Gamma$. There exists $m \geq 0$ such that $\tau^{m} M=\tau^{r} S$ and $\tau^{m} N=\tau^{s} S$ with $r, s \geq 0$. We may assume that $r \geq s$. By Lemma 2.1, $\operatorname{Hom}(M, N) \cong \operatorname{Hom}\left(\tau^{m} M, \tau^{m} N\right)=\operatorname{Hom}\left(\tau^{r} S, \tau^{s} S\right)$. Since $\left(\tau^{r} S\right)(a)=$ 0 , we see that $\tau^{r} S$ is a string representation; see [3, (5.19)]. Thus, $\tau^{r} S$ is a brick. Taking $N=M$, we see that $M$ is a brick. Suppose that $M \neq N$. Then $r>s$. Set $t=r-s$. Then $\Gamma$ has a sectional path $S_{t} \longrightarrow S_{t-1} \longrightarrow \cdots \longrightarrow S_{1} \longrightarrow \tau^{s} S$. For $x \in Q_{0}$, we have $\operatorname{dim} S_{t}(x)=\sum_{i=s}^{r} \operatorname{dim} \tau^{i} S(x)$. Since $\left(\tau^{i} S\right)(a)=0$ for $i \geq 0$, we obtain $\operatorname{dim} S_{t}(a)=0$. Hence, $S_{t}$ is a string representation; see [3, (5.19)]. If the supports of $\tau^{r} S$ and $\tau^{s} S$ have a common vertex $b$, then

$$
\operatorname{dim} S_{t}(b) \geq \operatorname{dim} \tau^{r} S(b)+\operatorname{dim} \tau^{s} S(b) \geq 2,
$$

contrary to $S_{t}$ being a string representation. Thus, $\tau^{r} S$ and $\tau^{s} S$ have disjoint supports. In particular, they are orthogonal, and so are $M$ and $N$. By Theorem 1.8 , $\Gamma$ is standard. In view of Statement (1), we have established Statement (2).

(3) Suppose that $Q$ is infinite but not of infinite Dynkin type. Then $Q$ has a finite subquiver $\Sigma$ of Euclidean type. Then we can find a homogeneous tube $\mathcal{T}$ in $\Gamma_{\operatorname{rep}^{b}(\Sigma)}$; see, for example, [3, (6.3)]. Let $M_{i}$ with $i \geq 1$ be the representations in $\mathcal{T}$ which are not quasi-simple. Regarded as representations of $Q$, the $M_{i}$ are distributed in infinitely many regular components of $\Gamma_{\mathrm{rep}^{+}(Q)}$; see [․ (6.1), (6.2)]. These regular components are not standard, since the $M_{i}$ have non-trivial endomorphism algebras. The proof of the theorem is completed.

Remark. (1) In view of Theorem 5.17 stated in $[3$, we see that wings and the translation quivers $\mathbb{Z} \mathbb{A}_{\infty}, \mathbb{N A}_{\infty}^{+}$and $\mathbb{N}^{-} \mathbb{A}_{\infty}^{-}$all occur as standard Auslander-Reiten components of Krull-Schmidt categories.

(2) Let $Q$ be finite of Euclidean type. If $k$ is not algebraically closed, then some indecomposable $k$-representations of $Q$ have a non-trivial automorphism field; see the proof in [3, (6.3)]. As a consequence, every connected component of $\Gamma_{\operatorname{rep}^{b}(Q)}$ is standard if and only if $k$ is algebraically closed. 
We conclude this section with an application to the bounded derived category $D^{b}\left(\operatorname{rep}^{+}(Q)\right)$ of $\operatorname{rep}^{+}(Q)$. Since $\operatorname{rep}^{+}(Q)$ is hereditary, the vertices of $\Gamma_{D^{b}\left(\mathrm{rep}^{+}(Q)\right)}$ can be chosen to be the shifts of those in $\Gamma_{\text {rep }}{ }^{+}(Q)$. If $Q$ is not of finite Dynkin type, then the connected components of $\Gamma_{D^{b}\left(\mathrm{rep}^{+}(Q)\right)}$ are the shifts of the regular components of $\Gamma_{\mathrm{rep}^{+}(Q)}$ and the shifts of the connecting component, which is obtained by gluing the preprojective component together with the shift by -1 of the preinjective components of $\Gamma_{\mathrm{rep}^{+}(Q)}$; see [5, (4.4)] and [3, (7.10)]. In case $Q$ is of finite Dynkin type, $\Gamma_{D^{b}\left(\mathrm{rep}^{+}(Q)\right)}$ is connected of shape $\mathbb{Z} Q^{\mathrm{op}}$, which is obtained by gluing, for each integer $i$, the shift by $i$ of $\Gamma_{\operatorname{rep}^{b}(Q)}$ together with its shift by $i+1$; see [5. (4.5)]. In this case, we also call $\Gamma_{D^{b}\left(\mathrm{rep}^{+}(Q)\right)}$ the connecting component.

2.3. Theorem. Let $Q$ be a connected quiver which is strongly locally finite.

(1) The connecting component of $\Gamma_{D^{b}\left(\mathrm{rep}^{+}(Q)\right)}$ is standard.

(2) If $Q$ is of finite or infinite Dynkin type, then every connected component of $\Gamma_{D^{b}\left(\mathrm{rep}^{+}(Q)\right)}$ is standard.

Proof. We denote by $\mathcal{C}_{Q}$ the connecting component of $\Gamma_{D^{b}\left(\mathrm{rep}^{+}(Q)\right)}$. Let $\Delta$ be the full subquiver of $\mathcal{C}_{Q}$ generated by the representations $P_{x} \in \Gamma_{\text {rep }^{+}(Q)}$ with $x \in Q_{0}$, which is isomorphic to $Q^{\mathrm{op}}$. It follows from Lemma 7.8 stated in 3 ] that $\Delta$ is a section of $\mathcal{C}_{Q}$. Since $\operatorname{rep}^{+}(Q)$ fully embeds in $D^{b}\left(\operatorname{rep}^{+}(Q)\right)$, by Theorem 2.2. $\Delta$ is standard. Let $M, N \in \operatorname{rep}^{+}(Q)$. Since $\operatorname{rep}^{+}(Q)$ is hereditary, $\operatorname{Hom}_{D^{b}\left(\mathrm{rep}^{+}(Q)\right)}(M[m], N[n])=0$ for $m>n$; see [7, (3.1)]. Combining this fact with the standardness of the preprojective component of $\Gamma_{\mathrm{rep}^{+}(Q)}$, we deduce that $\operatorname{Hom}_{D^{b}\left(\mathrm{rep}^{+}(Q)\right)}\left(\Delta^{+}, \Delta\right)=0$ and $\operatorname{Hom}_{D^{b}\left(\mathrm{rep}^{+}(Q)\right)}\left(\Delta \cup \Delta^{+}, \Delta^{-}\right)=0$.

If $Q$ is not of finite Dynkin type, then $\Delta^{+}$coincides with the full subquiver of the preprojective component of $\Gamma_{\text {rep }^{+}(Q)}$ generated by the non-projective representations, while $\Delta^{-}$coincides with the shift by -1 of the preinjective components of $\Gamma_{\text {rep }}{ }_{(Q)}$. Thus, $\Delta^{+}$contains no left infinite path and $\Delta^{-}$contains no right infinite path; see [3, (4.8)]. This is also the case if $Q$ is of finite Dynkin type; see (1.5). Thus $\mathcal{C}_{Q}$ is standard by Theorem 1.4. This establishes Statement (1). Combining this with Theorem $2.2(2)$, we obtain Statement (2). The proof of the theorem is completed.

Remark. Let $Q$ have no infinite path. If $Q$ is not of finite Dynkin type, then $\Gamma_{\text {rep }}{ }_{(Q)}$ has a unique preinjective component of shape $\mathbb{N} Q^{\text {op }}$ and its proprojective component is of shape $\mathbb{N}^{-} Q^{\mathrm{op}}$; see [3, (4.7)]. Thus the connecting component of $\Gamma_{D^{b}\left(\mathrm{rep}^{+}(Q)\right)}$ is always of shape $\mathbb{Z} Q^{\mathrm{op}}$.

\section{Specialization to module CAtegories of algebras}

Throughout this section, assume that $k$ is algebraically closed. Let $A$ stand for a finite-dimensional $k$-algebra and $\bmod A$ for the category of finite-dimensional left $A$-modules. In this classical situation, we have the following easy criteria for an Auslander-Reiten component with sections to be standard.

3.1. Theorem. Let $A$ be a finite-dimensional algebra over an algebraically closed field, and let $\Gamma$ be a connected component of $\Gamma_{\bmod A}$. If $\Delta$ is a section of $\Gamma$, then $\Gamma$ is standard if and only if $\operatorname{Hom}_{A}(\Delta, \tau \Delta)=0$, and if and only if $\operatorname{Hom}_{A}\left(\tau^{-} \Delta, \Delta\right)=0$. 
Proof. Let $\Delta$ be a section of $\Gamma$. Note that every module in $\Delta$ admits a proper sink map and a proper source map. Moreover, since the base field is algebraically closed, every module in $\Delta$ has a trivial automorphism field.

Suppose that $\operatorname{Hom}_{A}(\Delta, \tau \Delta)=0$. Then $\Delta$ is finite; see [14, (2.1)]. By Lemma 1.5. $\Delta^{+}$has no left infinite path and $\Delta^{-}$has no right infinite path. Assume that $\operatorname{Hom}_{A}(X, Y) \neq 0$ for some $X \in \Delta^{+}$and $Y \in \Delta^{-}$. Since every module in $\Delta^{+}$admits a sink epimorphism, we obtain an arrow $X_{1} \rightarrow X$ in $\Gamma$ such that $\operatorname{Hom}_{A}\left(X_{1}, Y\right) \neq 0$. Observe that $X_{1} \in \Delta \cup \Delta^{+}$. If $X_{1} \in \Delta^{+}$, then $\Gamma$ has an arrow $X_{2} \rightarrow X_{1}$ such that $\operatorname{Hom}_{A}\left(X_{2}, Y\right) \neq 0$. Since $\Delta^{+}$has no left infinite path, there exists a module $M$ in $\Delta$ such that $\operatorname{Hom}_{A}(M, Y) \neq 0$. Similarly, since $\Delta^{-}$has no right infinite path and every module in $\Delta^{-}$has a source monomorphism, there exists a module $N$ in $\tau \Delta$ such that $\operatorname{Hom}_{A}(M, N) \neq 0$, a contradiction. This shows that $\operatorname{Hom}_{A}\left(\Delta^{+}, \Delta^{-}\right)=0$. By Theorem 1.6. $\Gamma$ is standard. If $\operatorname{Hom}_{A}\left(\tau^{-} \Delta, \Delta\right)=0$, one shows in a dual manner that $\Gamma$ is standard. Conversely, it is evident that $\operatorname{Hom}_{A}(\Delta, \tau \Delta)=0$ and $\operatorname{Hom}_{A}\left(\tau^{-} \Delta, \Delta\right)=0$ if $\Gamma$ is standard. The proof of the theorem is completed.

Let $\Gamma$ be a connected component of $\Gamma_{\bmod A}$. Recall that $\Gamma$ is generalized standard if $\operatorname{rad}^{\infty}(\bmod A)$ vanishes in $\Gamma$; see [14]. It is known that $\Gamma$ is generalized standard if it is standard (see [9]), and the converse holds true in case $\Gamma$ has no projective module or no injective module; see [16]. Observing that the conditions on $\Delta$ stated in Theorem 3.1 are trivially verified in case $\Gamma$ is generalized standard, we obtain the following consequence.

3.2. Corollary. Let $\Gamma$ be a connected component of $\Gamma_{\bmod A}$. If $\Gamma$ has a section, then it is standard if and only if it is generalized standard.

The algebra $A$ is called tilted if $A=\operatorname{End}_{H}(T)$, where $H$ is a finite-dimensional hereditary algebra and $T$ is a tilting $H$-module. In this case, $\bmod A$ contains slices (see [6]), and a connected component of $\Gamma_{\bmod A}$ containing the indecomposable modules of a slice is called a connecting component. It is shown that a connecting component of a tilted algebra is standard; see [1, (5.7)].

3.3. Corollary. If $\Gamma$ is a connected component of $\Gamma_{\bmod A}$, then $\Gamma$ is standard with sections if and only if it is a connecting component of a tilted factor algebra of $A$.

Proof. Let $\Gamma$ be a connected component of $\Gamma_{\bmod A}$. Suppose first that $\Gamma$ is standard with a section $\Delta$. In particular, we have $\operatorname{Hom}_{A}(\Delta, \tau \Delta)=0$. If $I$ is the intersection of the annihilators of the modules in $\Gamma$, then $B=A / I$ is a tilted algebra with $\Gamma$ a connecting component of $\Gamma_{\bmod B}$; see [8, (2.2)] and also [15].

Suppose next that there exists a tilted algebra $B=A / I$ with $\Gamma$ being a connecting component of $\Gamma_{\bmod B}$. Then $\Gamma$ has a section $\Delta$ generated by the non-isomorphic indecomposable modules of a slice of $\bmod B$. By the defining property of a slice, $\operatorname{Hom}_{B}(\Delta, \tau \Delta)=0$. Thus, by Theorem 3.1 $\Gamma$ is a standard component of $\Gamma_{\bmod B}$. Since $\bmod B$ fully embeds in $\bmod A$, we see that $\Gamma$ is a standard component of $\Gamma_{\bmod A}$. The proof of the corollary is completed.

\section{REFERENCES}

[1] Ibrahim Assem, Juan Carlos Bustamante, and Patrick Le Meur, Coverings of laura algebras: the standard case, J. Algebra 323 (2010), no. 1, 83-120, DOI 10.1016/j.jalgebra.2009.08.013. MR2564830(2011e:16017)

[2] Raymundo Bautista, Irreducible morphisms and the radical of a category, An. Inst. Mat. Univ. Nac. Autónoma México 22 (1982), 83-135 (1983). MR736555 (86g:16041) 
[3] Raymundo Bautista, Shiping Liu, and Charles Paquette, Representation theory of strongly locally finite quivers, Proc. London Math. Soc. 106 (2013), 97-162. MR3020740

[4] K. Bongartz and P. Gabriel, Covering spaces in representation-theory, Invent. Math. 65 (1981/82), no. 3, 331-378, DOI 10.1007/BF01396624. MR643558(84i:16030)

[5] Dieter Happel, On the derived category of a finite-dimensional algebra, Comment. Math. Helv. 62 (1987), no. 3, 339-389, DOI 10.1007/BF02564452. MR910167 (89c:16029)

[6] Dieter Happel and Claus Michael Ringel, Tilted algebras, Trans. Amer. Math. Soc. 274 (1982), no. 2, 399-443, DOI 10.2307/1999116. MR675063 (84d:16027)

[7] Helmut Lenzing, Hereditary categories, Handbook of tilting theory, London Math. Soc. Lecture Note Ser., vol. 332, Cambridge Univ. Press, Cambridge, 2007, pp. 105-146, DOI 10.1017/CBO9780511735134.006. MR2384609 (2009c:18015)

[8] Shiping Liu, Tilted algebras and generalized standard Auslander-Reiten components, Arch. Math. (Basel) 61 (1993), no. 1, 12-19, DOI 10.1007/BF01258050. MR1222084 (94g:16017)

[9] Shiping Liu, Infinite radicals in standard Auslander-Reiten components, J. Algebra 166 (1994), no. 2, 245-254, DOI 10.1006/jabr.1994.1150. MR.1279256 (95g:16014)

[10] Shiping Liu, Shapes of connected components of the Auslander-Reiten quivers of Artin algebras, Representation theory of algebras and related topics (Mexico City, 1994), CMS Conf. Proc., vol. 19, Amer. Math. Soc., Providence, RI, 1996, pp. 109-137. MR1388561 (97e:16037)

[11] Shiping Liu, Auslander-Reiten theory in a Krull-Schmidt category, São Paulo J. Math. Sci. 4 (2010), no. 3, 425-472. MR2856194

[12] Claus Michael Ringel, Finite dimensional hereditary algebras of wild representation type, Math. Z. 161 (1978), no. 3, 235-255, DOI 10.1007/BF01214506. MR501169 (80c:16017)

[13] Claus Michael Ringel, Tame algebras and integral quadratic forms, Lecture Notes in Mathematics, vol. 1099, Springer-Verlag, Berlin, 1984. MR774589 (87f:16027)

[14] Andrzej Skowroński, Generalized standard Auslander-Reiten components, J. Math. Soc. Japan 46 (1994), no. 3, 517-543, DOI 10.2969/jmsj/04630517. MR1276836 (95d:16022)

[15] Andrzej Skowroński, Generalized standard Auslander-Reiten components without oriented cycles, Osaka J. Math. 30 (1993), no. 3, 515-527. MR.1240010(94k:16026)

[16] Andrzej Skowroński, On semi-regular Auslander-Reiten components, Bull. Polish Acad. Sci. Math. 42 (1994), no. 2, 157-163. MR.1810701

Département de Mathématiques, Université de Sherbrooke, Sherbrooke, Québec J1K 2R1, CANADA

E-mail address: shiping.liu@usherbrooke.ca

Department of Mathematics and Statistics, University of New Brunswick, FrederiCton, New Brunswick E3B 5A3, CANAdA

E-mail address: charles.paquette@usherbrooke.ca 